\title{
Tipps für die Abrechnung indizierter und nicht indizierter Behandlungsleistungen \\ (1)
}

\author{
Dr. Gwendolyn Gemke, Fachanwältin für Medizinrecht, München
}

Immer wieder verweigern private Krankenversicherer die Erstattung von Behandlungsleistungen unter Berufung auf eine angeblich fehlerhafte Rechnungsstellung. Neuerdings wird immer wieder dann die Rechnungstellung moniert, wenn in einer Rechnung sowohl medizinisch indizierte, als auch nicht indizierte Leistungen abgerechnet werden. Bei der pauschalen Verweigerung der Erstattung durch die Versicherer bleiben die rechtlichen Anforderungen an eine ordnungsgemäße Abrechnung häufig unberücksichtigt. Recht und Wirklichkeit fallen auseinander.

Unabhängig von ihrer medizinischen Indikation ist für die Abrechnung ärztlicher Leistungen die Gebührenordnung für Ärzte (GOÄ) verbindlich.

Eine rechtliche Verpflichtung des Arztes, medizinisch indizierte Leistungen und nicht indizierte in getrennten Rechnungen abzurechnen, besteht gerade nicht. Folglich liegt in der gemeinsamen Abrechnung auch kein Abrechnungsfehler, so dass mit Rechnungsstellung die Zahlungspflicht des Patienten begründet wird.

\section{Übermaßleistungen müssen gekennzeichnet werden}

Allerdings ist der Arzt gemäß $\$ 12$ Abs. 3 Satz 5 GOÄ verpflichtet, diejenigen Leistungen, die nicht medizinisch notwendig sind und dennoch auf Verlangen des $\mathrm{Pa}$ tienten erbracht wurden (sog. Übermaßleistungen) als solche in der Rechnung zu kennzeichnen. De facto besteht also eine Pflicht zur Differenzierung zwischen den beiden Leistungsarten.

Was bedeutet dies für die Praxis? Letztendlich bleiben dem Arzt zwei Möglichkeiten der Rechnungsstellung:

Zum einen können im Rahmen einer einheitlichen Rechnung die medizinisch nicht notwendigen - und damit auch die nicht indizierten Leistungen - abgerechnet werden, sie sind dann aber deutlich zu kennzeichnen. Den Patienten wird diese Kennzeichnung nicht erfreuen, da sich dadurch in Grenzfällen seine Chancen auf eine Erstattung durch die private Krankenversicherung zerschlagen. Allerdings ist dies bei den Leistungen, die ohne medizinische Indikation erbracht werden, auch richtig so. Oft hilft ein Wechsel der Perspektive - schließlich steigt damit die Chance des Patienten, die ebenfalls in der Rechnung enthaltenen notwendigen Leistungen zügig erstattet zu erhalten.

\section{Rückfragen verursachen erheblichen Aufwand}

Dies liegt auch im Interesse des abrechnenden Arztes, denn Rückfragen der Versicherer oder der Patienten verursachen einen erheblichen Aufwand und häufig zahlen die Patienten erst dann, wenn die Krankenkasse erstattet hat.

Zum anderen können zwei getrennte Rechnungen gestellt werden, so dass für Patienten und Versicherer auf einen Blick erkennbar ist, um welche Leistungen es sich jeweils handelt und in welcher Höhe ein Erstattungsanspruch gegen die Krankenversicherung besteht - bzw. in welcher Höhe der Patient selbst in die Tasche greifen muss.

Soweit der damit einhergehende höhere Verwaltungsaufwand gescheut wird, muss auf diese Abrechnungsvariante jedoch spätestens dann zurückgegriffen werden, wenn Leistungen erbracht werden, die umsatzsteuerpflichtig sind. Beispielhaft seien hier kosmetische Leistungen genannt. Über diese umsatzsteuerpflichtigen Leistungen ist eine gesonderte Rechnung - ebenfalls nach GOÄ und unter Ausweis der Umsatzsteuer - zu stellen.

Gründe genug die Form der Rechnungsstellung zu überdenken. Für wel-
Welche Leistungen müssen wie abgerechnet werden?

che Variante man sich entscheidet, ist letztlich Geschmackssache. Zu beachten ist aber, dass die Kennzeichnung der Übermaßleistungen gesetzlich vorgeschrieben ist.

\section{Langfristig sparen Sie Kosten und Zeit}

Diese Kennzeichnung kann, ähnlich wie bei der Abrechnung von Analogleistungen, durch einen kurzen Hinweis auf $\$ 12$ Abs. 3 Satz 5 GOÄ oder den Zusatz "auf Verlangen" in der Leistungsbezeichnung erfolgen.

Beide Rechnungsvarianten führen zwar eventuell kurzfristig zu Unmut beim Patienten, tragen jedoch langfristig zur Vermeidung von kosten- und zeitintensiven Auseinandersetzungen bei der Einforderung des eigenen Honorars bei. 